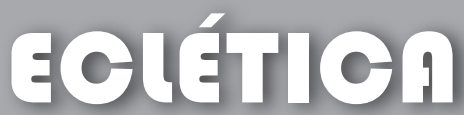 química
}

\section{DETERMINAÇÃO COLORIMÉTRICA DE METABISSULFITO}

\author{
Humberto da Silva Junior; Gutto Raffyson Silva de Freitas; Francisco Régis da Silva Pereira; Robson \\ Fernandes de Farias; Francisco Claudece Pereira*. \\ Departamento de Química - Universidade Federal do Rio Grande do Norte UFRN, Brasil \\ C P 1524, 59072-970, Natal-RN, Brasil. \\ (*)E-mail: claudece@ufrnet.br
}

Resumo: O ânion metabissulfito é largamente usado como agente anti-oxidante e preservativo de uma variedade de produtos industrializados. A interação desta espécie com o corante pararosanilina é capaz de promover o rebaixamento da banda de absorção do pigmento na região do visível. Utilizando as melhores condições experimentais, tais como, solução tampão Britton e Robinson, pH 5,0 e corante em $1,0 \times 10^{-4} \mathrm{~mol} \mathrm{~L}^{-1}$, foi possível obter uma relação entre concentração de metabissulfito e absorbância, na faixa entre $1,3 \times 10^{-6} \mathrm{~mol} \mathrm{~L}^{-1}$ a $3,0 \times 10^{-5} \mathrm{~mol} \mathrm{~L}^{-1}$. O limite

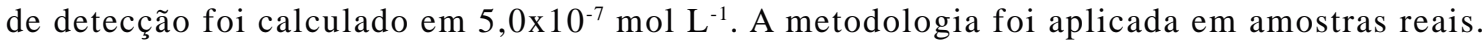

Palavras-chave: metabissulfito, pararosanilina, espectrofotometria.

\section{Introdução}

Alguns compostos sulfurados são largamente empregados em distintas áreas da cadeia produtiva, com ação preservativa e anti-oxidante em bebidas do tipo vinhos, cidra, champanhes e cervejas. Também encontra larga aplicação na prevenção do enegrecimento de vegetais e frutas e muito usado para manter as características de abricós, abacate, beterraba, uva, limão, cogumelo, batatas, dentre outros. De forma similar é utilizado como fonte de dióxido de enxofre em formulações farmacêuticas como anestésicos, colírios, soluções tópicas, anti-micótico, nutrição parenteral e injeções. Aplicado nas indústrias de cosméticos, fotográfica e alimentícia; nesta ultima, atuando como inibidor microbial, contra leveduras e bactérias em produtos como gelatinas, frutas desidratadas, biscoitos, chocolates, alimentos a base de amido, passas, salames, lingüiça, camarão fresco, etc. Recentemente, algumas substâncias desta ca- tegoria têm encontrado aplicação como aditivos em preparações minerais e vitaminas [1-4].

Diferentes compostos desta classe têm sido definidos como precursores de $\mathrm{SO}_{2}$ e, assim, os teores ingeridos destas espécies, expressos como equivalentes de dióxido de enxofre. Uma avaliação da Federation of American Societies for Experimental Biology (FASEB) estima que teores entre 30-100 mg SO não apresentam efeitos adversos aos seres humanos. Em contrapartida, a Organização Mundial da Saúde (OMS) estabelece como aceitável uma ingestão diária de compostos sulfetados na ordem de $0,7 \mathrm{mg} \mathrm{kg}^{-1}$ expresso como $\mathrm{SO}_{2}$. Em indivíduos normais, os teores de sulfitos presentes no soro alcançam limites de 4 a $5 \mathrm{nmol}$ $\mathrm{L}^{-1}$. A enzima mitocondrial sulfito oxidase é a responsável pela oxidação do $\mathrm{SO}_{2}$ a sulfato, que é excretado pela urina [5].

Dentre estes agentes, o metabissulfito (MBS) é um pó branco e cristalino, com odor pungente e caracteriza-se pela sua ação tóxica que 
inclui desordem no anticorpo imunoglobulina e processos de dermatites [6-7].

MBS apresenta os mesmos efeitos citotóxicos e genotóxicos observados para o dióxido de enxofre, uma vez que é responsável por aberraç̃os crosson ções crom de está relacionada a perturbaçôs celulares, comprometima na peroxidação de lipídeos. Em adição, pessoas com alergias e tendências asmáticas devem evitar ingerir alimentos aditivados com MBS e seus precursores, uma vez que MBS induz a bronco constrição. Numerosos efeitos adversos têm sido atribuídos aos agentes sulfetados, incluindo sintomas de urticárias, pruridos, angiodemas, asmas, dores de cabeça, lesões cutâneas, etc., [1, 4, 8-11].

Sais de MBS e correlatos são considerados tóxicos a determinados microorganismos, tais como, fungos e bactérias e, esta propriedade, explorada em diversas áreas a fim de combater estes agentes microbiológicos. Dentre estes, a Erwinia carotovora subsp. Atroseptica e Erwinia carotovora subsp. carotovora são responsáveis por consideráveis perdas econômicas na agricultura. O mecanismo de ação destas instituições químicas relaciona-se com a inativação de sistemas enzimáticos nos microorganismos, ju tatente commáticos nos microorganisnos, juntaninene c co-fator tianina pirofosfato e a co-nzina NAD. Este efeito é cap biológicas [12].

De acordo com a literatura o metabissulfito $\left(\mathrm{S}_{2} \mathrm{O}_{5}^{-2}\right)$ é prontamente convertido a bissulfito $\left(\mathrm{HSO}_{3}^{-}\right)$em contato com a água $[3,5,8]$. Sob a ação do oxigênio, soluções de MBS são facilmente transformados em outras espécies, tais como, sulfitos, sulfatos, sulfetos e tiossulfatos. Assim, distintas espécies sulfetadas podem estar presentes em matrizes comerciais como subproduto ou produto de decomposição [1].

Métodos analíticos para a determinação de oxiânions de enxofre têm sido baseados em técnicas amperométricas [13], quimiluminescência [14], eletroforese capilar [15], polarográficas [16], titulações iodométricas [17], potenciométricas [18] e cromatografia iônica [19]. De uma forma geral, as metodologias empregam sofisticados equipamentos, tratamento prolongado dos padrões e das amostras e não são adequados para aplicações rotineiras [1].
De acordo com a literatura, o corante pararosanilina $(\mathrm{P})$ tem encontrado largo emprego $\mathrm{n}$ análise quantitativa das mais variadas espécies, ais como, $\mathrm{SO}_{2}$ [20-25], alguns compostos organicos [26-37] e espécies como bromato [38, 39] brometo [40] e alguns metais [41, 42].

Diante do exposto e, considerando a imporância no desenvolvimento de métodos analítico capazes de monitorar MBS, o presente trabalho exibe uma nova sistemática baseada na reação do MBS com o corante P (cloridrato de benzenoamina 4-(4-aminofenil)(4-imino-2,5-ciclohexadiano-1-ilideno)-metil; também conhecido como paramagenta, magenta ou fucsina básica (CAS 569-61-9), cuja formula é mostrada na Figura 1. A metodologia permite a determinaça espectroA eletromagnético.

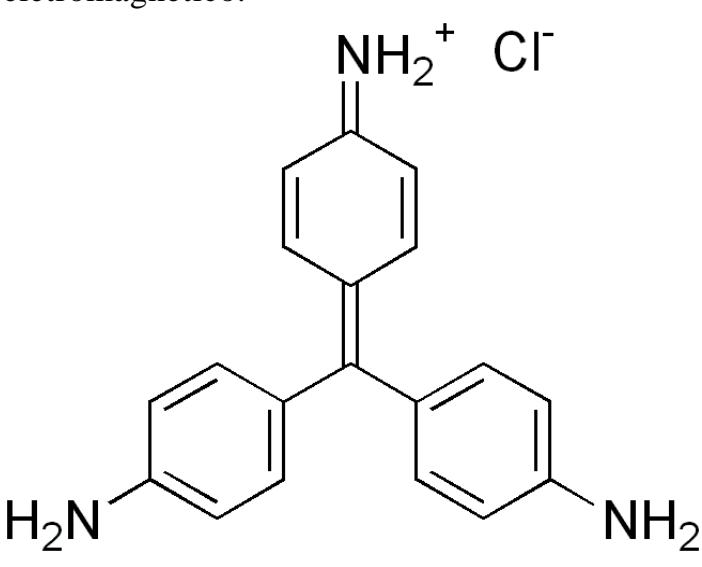

Figura 1. Fórmula estrutural da pararosanilina.

\section{Materiais e métodos}

\section{Equipamentos}

Espectrofotômetro UV-VIS 1650 PC da marca Shimadzu. Todos os experimentos foram conduzidos com cubetas de quartzo de $1 \mathrm{~cm}$ de caminho ótico. Para a preparação das soluções de trabalho foi empregada uma balança analítica da marca Marte Mict. Os valores de pH das soluções foram ajustados com um pHmetro da marca Mar- coni PA200, modelo RS232. Antes de seu uso, o eletrodo combinando de vidro foi previamente calibrado com as soluções fornecidas pelo fabricante.

Reagentes

Metabissulfito de sódio (Merck), corante ararosanilina (Merck), ácido acético (Vtec), ácido fosfórico (Merck), ácido bórico (Merck) e hidróxido de sódio (Merck). Todos os reagentes de grau analítico.

\section{Procedimentos experimentais}

Soluções tampão Britton e Robinson (BR) foram obtidas através da mistura dos ácidos acético, fosfórico e bórico. $\mathrm{O}$ pH das soluções, no intervalo de 2 a 12, foi ajustado pela adição de hidróxido de sódio. Soluções do corante e metabissulfito de sódio foram obtidas através da dissolução dos sais diretamente em água. Todas as sluções foram preparas utilizando água bideságua bidestilado.

Duas matrizes foram analisadas: um espumante do tipo sidra Cereser, facilmente encontrado em qualquer supermercado do país e amostras coletadas nos tanques de cultivo de camarão (carcinicultura) existentes no Estado do Rio Grande do Norte. Alíquotas das amostras foram adicionas a balões volumétricos contendo $1 \times 10^{-4} \mathrm{~mol} \mathrm{~L}^{-1} \mathrm{do}$ corante, em solução tampão B-R pH 5,0, e analisadas diretamente sem qualquer tratamento.

\section{Resultados e discussão}

O comportamento espectrofotométrico do ânion MBS foi investigado em amplo intervalo de pH $(2,0$ a 12,0$)$ em solução tampão B-R. O analito apresenta um único e bem definido pico em 277 nm (em pH 2,0) que aumenta em função dos incrementos de MBS (Figura 2A). Com o aumento nos valores de $\mathrm{pH}$ observa-se uma vertiginosa queda do sinal a partir de $\mathrm{pH} 2,0$, com total

Ecl. Quím., São Paulo, 35 - 3: 133 - 139, 2010 supressão em $\mathrm{pH} \geq 4,0$ (Figura 2B). Em adição, o monitoramento direto da espécie somente pode ser realizado em altos valores de concentração.

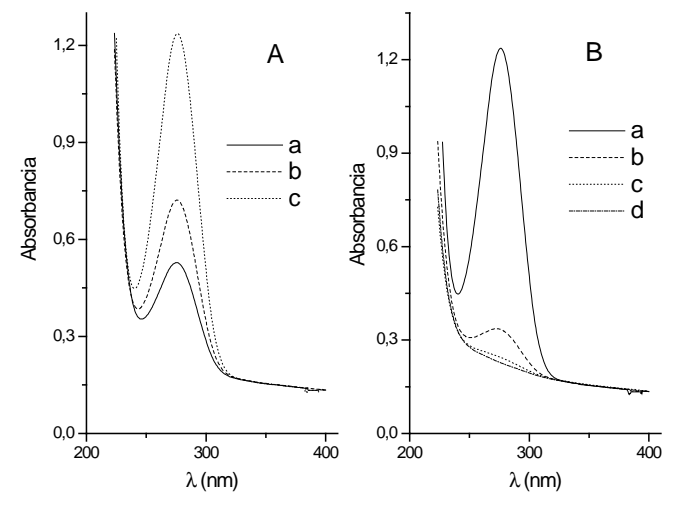

Figura 2A. Espectros de absorção molecular de MBS em solução tampão B-R pH 2,0. Curvas: (a) (c) $\mathrm{MBS}=5,0 \times 10^{-3} \mathrm{~mol} \mathrm{~L}^{-1}$. Figura $2 \mathrm{~B}$ : Efeito (c) MBS $=5,0 \times 10^{-3} \mathrm{~mol} \mathrm{~L}^{-1}$. Figura 2B: Efeito do $\mathrm{pH}$ B-R. Curvas: (a) $\mathrm{pH}_{2}, 0$; (b) $\mathrm{pH} 3,0$; (c) $\mathrm{pH} 4,0$ (d) pH 5,0.

Por outro lado, soluções de $5 \times 10^{-5} \mathrm{~mol} \mathrm{~L}^{-1}$ do corante $\mathrm{P}$ exibem três bandas de absorção centradas em 1545 nm (pico A), 1 290nm (pico B) e $1248 \mathrm{~nm}$ (рісо C) (Figura 3). O pico da região do visível é extremamente dependente das variações de $\mathrm{pH}$. O sinal permanece ligeiramente constante em valores de $2,0 \leq \mathrm{pH} \leq 5,0$, seguindo de acentuado decréscimo e extinção em $\mathrm{pH} \geq 8,0$. Este comportamento está associado a desprotonação da estrutura quinoidal de $\mathrm{P}$, e o deslocamento da dupla ligação do carbono central, a fim de recompor o sistema do corante (Figura 1) [43]. 


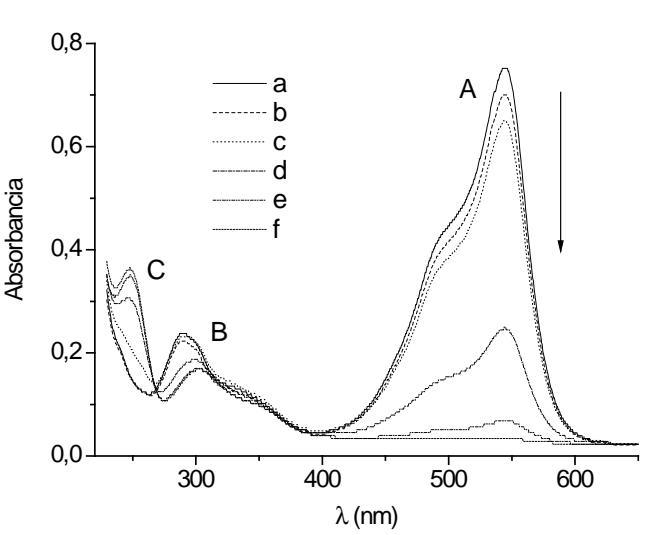

Figura 3. Espectros de absorção UV-Vis de $5 \times 10^{-5}$ mol L-1 de P em solução tampão B-R. Curvas: (a) pH 3,0; (b) $\mathrm{pH} \mathrm{4,0;} \mathrm{(c)} \mathrm{pH} \mathrm{5,0;} \mathrm{(d)} \mathrm{pH} 6,0$; (e) $\mathrm{pH} 7,0$ e (f) $\mathrm{pH} 8,0$.

A seguir avaliou-se o efeito da mistura do corante P com o MBS em solução tampão B-R pH 4,0. Para este estudo fixou-se a concentração de $P$ em $8 \times 10^{-5} \mathrm{~mol} \mathrm{~L}^{-1} \mathrm{e}$ adições de MBS no intervalo de concentração entre $5 \times 10^{-5}$ a $5 \times 10^{-4} \mathrm{~mol} \mathrm{~L}^{-1}$. Os resultados são mostrados na Figura 4.

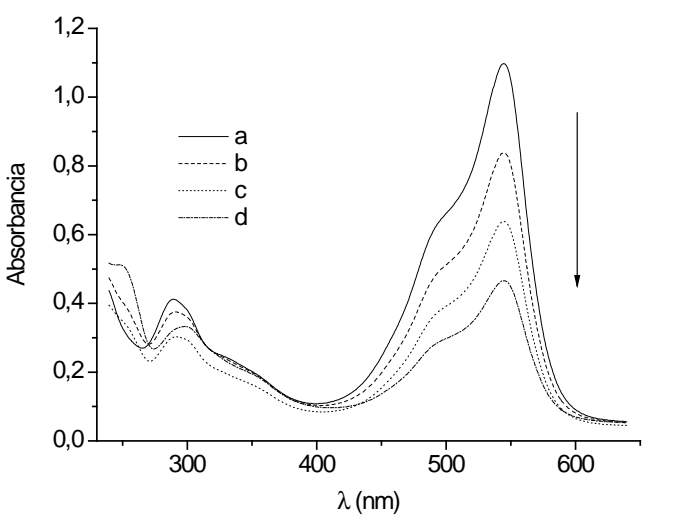

Figura 4. Espectros de absorção UV-Vis para a mistura de $8 \times 10^{-5} \mathrm{~mol} \mathrm{~L}^{-1}$ de P com MBS em solução tampão B-R pH 4,0. Curvas: (a) 0,0 de MBS; (b) adição de $5 \times 10^{-5} \mathrm{~mol} \mathrm{~L}^{-1}$ de MBS; (c) adição de $1 \times 10^{-4} \mathrm{~mol} \mathrm{~L}^{-1}$ de MBS e (d) adição de $5 \times 10^{-4} \mathrm{~mol}$ $\mathrm{L}^{-1}$ de MBS.
Os espectros mostram que, a banda correspondente a absorção da função quinoidal de $\mathrm{P}$, diminui sua altura em função dos incrementos na concentração de MBS.

De acordo com a literatura, a interação de substâncias sulfuradas como o corante P, ocorre por uma reação de adição, no qual, a dupla ligação quinoidal do corante, sofre uma cisão e incorpora a espécie inorgânica através da ligação com o átomo de enxofre no carbono central de $\mathrm{P}$, provocando a descoloração do pigmento [44, 45].

$\mathrm{O}$ efeito da mudança nos valores de $\mathrm{pH}$, sobre o sinal da mistura de $\mathrm{P}$ com MBS, foi avaliado mantendo-se a concentração de $\mathrm{P}$ fixa em $8 \times 10^{-5}$ mol L-10 adiços de MBS no intervalo de con-

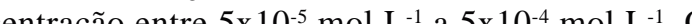
estudo foi conduzido em solução tampão B-R e os resultados mostrados na Figura 5.

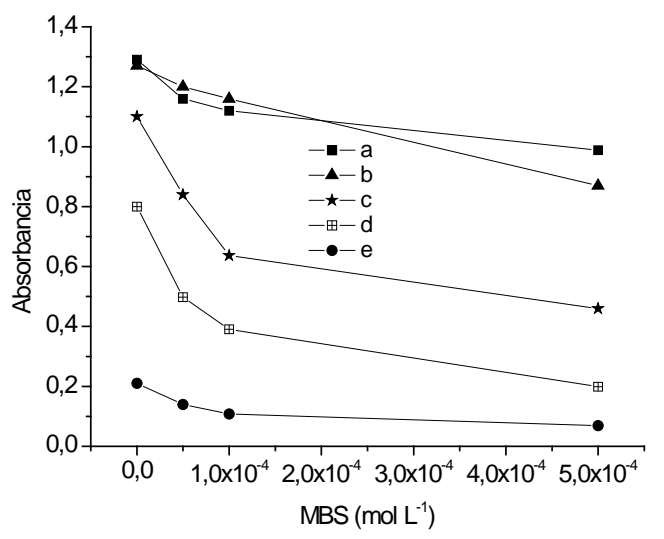
Figura 5. Efeito do $\mathrm{pH}$ sobre o sinal de absorbância de MBS no interva $8 \times 10^{-5} \mathrm{~mol} \mathrm{~L}^{-1}$ de $\mathrm{P}$ e adiçoes solucão tampão B-R Curva: (a) $\mathrm{pH}$ 20; (b) $\mathrm{pH} 3,0$

(c) $\mathrm{pH} 4,0^{\circ}$ (d) $\mathrm{pH} 5,0$ e (e) $\mathrm{pH} 6,0$

Em todos os valores de $\mathrm{pH}$ investigados, a adição de MBS provoca a queda do sinal do pigmento a partir da primeira alíquota de MBS Adições posteriores do analito confirmam o decaimento do sinal do corante.

$$
\text { A influencia no aumento na concentração }
$$
de MBS sobre 0 sinal de $P$ foi avaliado em valores de $\mathrm{pH}$ 4,0 e 5,0 em distintas concentrações do pigmento. Em pH 4,0, independentemente da con- centração fixa de P utilizada, a curva de absorbância em função da crescente concentração de MBS, apresenta uma resposta de decaimento de segunda ordem, mostrando comportamento ano

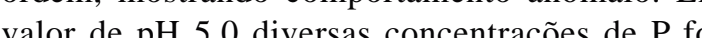
alor possível a construção de uma curva analítica par MBS, de acordo com a Figura 6.

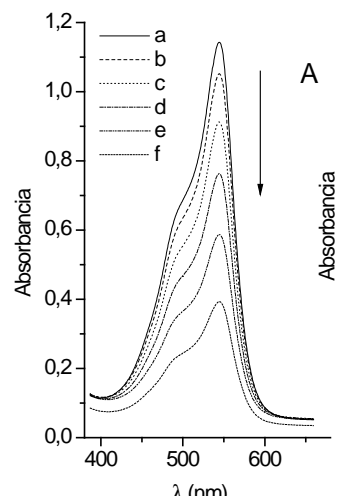

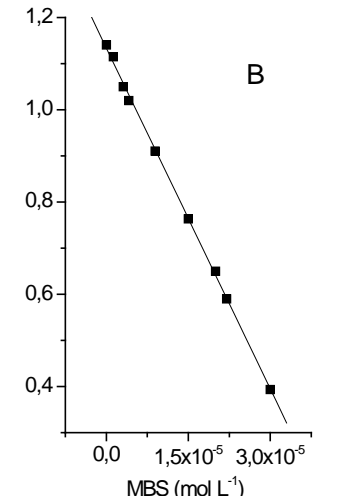

MBS (mol $\left.L^{-1}\right)$
Figura 6A. Espectros de absorção UV-Vis da mistura $1 \times 10^{-4} \mathrm{~mol} \mathrm{~L}^{-1}$ de $\mathrm{P}$ em tampão B-R pH 5,0 e adições de MBS. Curvas: (a) 0,0 de MBS, (b) 3,1 $1 \times 10^{-6} \mathrm{~mol}$ $\mathrm{L}^{-1}$ de MBS, (c) $9,2 \times 10^{-6} \mathrm{~mol} \mathrm{~L}^{-1}$ de MBS, (d) $1,5 \times 10^{-}$ ${ }^{5} \mathrm{~mol} \mathrm{~L}^{-1}$ de MBS, (e) 2,2x10-5 $\mathrm{mol} \mathrm{L}^{-1}$ de MBS e (f) $3,0 \times 10^{-5} \mathrm{~mol} \mathrm{~L}^{-1}$ de MBS. Figura 6B: Curva analítica (anse de análise $d e$ MBS, foi estabelecida quando se fixa a concentração de P em $1 \times 10^{-4} \mathrm{~mol} \mathrm{~L}^{-1}$. Nestas condições, fo

Tabela 1. Valores de concentração de MBS encontrados nas amostras analisadas.

\begin{tabular}{ccc}
\hline Amostra & MBS $\left(\mathrm{mol} \mathrm{L}^{-1}\right)$ & $\begin{array}{c}\text { Desvio-padrão }(*) \\
{[46,47]}\end{array}$ \\
\hline Espumante (Sidra) & $3,82 \times 10^{-4}$ & $\pm 0,25$ \\
Águas de criação de camarão & $8,18 \times 10^{-5}$ & $\pm 0,22$ \\
\hline
\end{tabular}

\section{$(*) \mathrm{n}=5$ medidas.}

\section{Conclusões}

A interação do MBS com o corante pararosanilina permite o monitoramento espectrofotométrico deste ânion, em matrizes que apresentam relativa complexidade, devido o grande número de constituintes existentes nestes meios. Nenhu- para MBS obtida a partir de 6A.

Os espectros da Figura 6A mostram que pico da região do visível, correspondente a absorção de $\mathrm{P}$, decresce à medida que aumenta concentração de MBS. Uma relação linear foi observada em toda a faixa de concentração de MBS investigada, expressa pela relação:

$\mathrm{Y}=1,13-2,46 \times 10^{4}[\mathrm{MBS}], \mathrm{R}=0,9995, \mathrm{n}=9$

onde $[\mathrm{MBS}]=$ concentração de MBS (mol $\left.\mathrm{L}^{-1}\right), \mathrm{R}=$ coeficiente de correlação e $\mathrm{n}=$ número de medidas. O limite de detecção foi estimado em $5 \times 10^{-7} \mathrm{~mol} \mathrm{~L}^{-1}$ através da relação sinal ruído $(\mathrm{n}=5)$ $[46,47]$.

A metodologia foi testada quanto a possíveis interferentes. Para uma concentração fixa do corante em $1 \times 10^{-4} \mathrm{~mol} \mathrm{~L}^{-1}$ em tampão B-R pH 5,0 , foram adicionadas alíquotas de $1 \times 10^{-4} \mathrm{~mol}$ $\mathrm{Cl}^{-}, \mathrm{Br}^{-1} \mathrm{SO}^{-2}$ e SCN $\mathrm{S}^{-}$Nenhuma interferência foi observada para estas condições experimentais.

A aplicação do método foi realizada em amostras de espumante (champanhe) comercial e águas de tanques de criação de camarão. Utilizando a equação da curva analítica em (I), em conjunto com as melhores condições experimentais, foi possível obter os resultados mostrados na Tabela 1.

Ecl. Quím., São Paulo, 35 - 3: 133 - 139, 2010 ma interferência foi constatada nas amostras estudadas, e as medidas podem ser realizadas sem a necessidade de tratamentos prévios. MBS pode ser detectado em valores de concentração tão baixo quanto $5 \times 10^{-7} \mathrm{~mol} \mathrm{~L}^{-1}$, em solução tampão B-R, em valor de $\mathrm{pH} 5,0$ na presença de $1 \times 10^{-4} \mathrm{~mol} \mathrm{~L}^{-1}$ de $\mathrm{P}$, como melhores condições de trabalho. 
Abstract: Metabisulfite anion is widely used as an antioxidant agent and preservative of a number of industrialized products. The interaction of this agent with pararosaniline dye can reduce the absorption band of the pigment within the visible area. From the best experimental conditions such as Britton-Robinson buffer solution, $\mathrm{pH}$ 5.0, and dye in a solution of $1.0 \times 10^{-4}$ $\mathrm{mol} \mathrm{L}^{-1}$, it was possible to obtain a relation between concentration of metabisulfite and absorin $5.0 \times 10^{-7} \mathrm{~mol} \mathrm{~L}^{-1}$. The methodology was applied on real samples.

\section{Keywords: Metabisulfite, Pararosaniline, Spectrophotometry.}

\section{Referências}

[1] M. Novakova, L. Krivankova, M. Bartos, V. Urbanova, K. Vytras, Talanta, 74 (2007) 183.

[2] F. N. A. Lopez, J. B. Gallego, M. C. D. Quintana, A. G. Fernandez, LWT, 41 (2008) 551.

3] V. Madan, S. L. Walker, M. H. Beck, Contact Dermatitis, 57, (2007) 173

4] M. Steiner, A. Scaife, S. Semple, G. Hulks, J. G. Ayres, Occupational Medicine, 58 (2008) 545.

[5] O. Elmas, M. Aslan, S. Caglar, N. Derin, A. Agar, Y. Alıcıguzel, P. Yargıçoglu, Regulatory Toxicology and Pharmacology, 42 (2005) 77.

[6] L. Stingeni, L. Bianchi, P. Lisi, Contact Dermatitis, 60 (2009) 52.

[7] B. Jankovic, S. Mentus, M. Jankovic, Journal of Physics and Chemistry of Solids, 69 (2008) 1923.

(8) S. Heshmati, H. I. Maibach, Contact Dermatitis, 41 (1999) 166

[9] A. Kayraldiz, M. Topaktas, Russian Journal of Genetics, 43(8) (2007) 905.

[10] E. Rencüzogullari, H. B. Plla, A. Kayraldiz, M. Topaktas, Mutation Research, 490 (2001) 107.

[11] T. J. Avis, M. Michaud, R. J. Tweddell, Applied and Environmental Microbiology, 73(9) (2007) 2820.

[12] E. S. Yaganza, D. Rioux, M. Simard, J. Arul, R. J. Tweddell, Applied and Environmental Microbiology, 70(11) (2004) 6800.

[13] M. S. M. Quintino, K. Araki, H. E. Toma, L. Angnes, Talanta, 68 (2006) 1281

[14] D. Y. He, Z. J. Zhang, Y. Huang, Anal. Lett. 38 (2005) 563.

[15] G. Jankovskiene, Z. Daunoravicius, A. Padarauskas, J. Chromatogr. A, 934 (2001) 67.

[16] M. L. Carvalho, G. Schwedt, Anal. Chim. Acta, 436 (2001) 293.

L. Brown, L. Szekeres, Talanta, 26 (1979) 414

[18] B. Meyer, M. Ospina, L. P. Peter, Anal. Chim. Acta, 117 (1980) 301.

[19] P. L. Hildmi, S. Cavalli, A. Trifiro, J. Chromatogr. 789

20] M. Kass, A. Ivaska, Analytica Chim. Acta. 449 (2001)

[21] M. A. Segundo, A. O. S. S. Rangel, Analytica Chim. Acta, 427 (2001) 279.

[22] M. A. Segundo, A. O. S. S. Rangel, A. Cladera, V. Cerda, Analyst, 125 (2000) 1501

[23] H. Vekisquez, H. Ramfrez, J. Dfaz, M. G. Nava, B. S, Borrego, J. Morales, Journal of Chromatography A, 739 (1996) 295.

[24] K. Irgum, Anal. Chem. 57 (1985) 1335.

[25] S. K. Goyal, Environmental Monitoring and Assessment, 20 (2006) 461.

[26] M. Wahbi, H. Abdine, M. A. Koranyand, M. H. A. Hay, Analyst, 103 (1978) 876.

27] M. C. Wu, C. M. Jiang, Y. Y. Ho, S. C. Shen, H. M. Chang, Food Chemistry, 100 (2007) 412.

J. M. B. Sendra, S. Pescarolo, L. C. Rodríguez, A. M. G. Campaña, E. M. A. López, Fresenius J. Anal. Chem. 369 (2001) 715.

[29] L. G. Gracia, M. D. L. Castro, Analyst, 124 (1999) 1119 [30 W. J. Groah, J. Bradfleld, G. Gramp, R. Rudzinski, G. (31) P. E. Gen. Sci. Technol. 25 (1991) 117.

[3] P. E. Georghiou, L. Harlick, L. Winsor, D. Snow, (32) R. R. Misch, D. W. A

L. Z. Fanning, C. D Ganvllle, Anal. Chem. 53 (1981)

[33] L. T. Gibsona W. J. Kerra, A. Nordona, J. Reglinski C. Robertson, L. Tunbull, C. M. Watt, A. Chengh, W. C.

ma, S. Motomizu, 35] Y. Y. Maru, J. Nakn 165.

.

zumi, Sensors and Actuators B, 129 (2008) 544.
36] S. K. Dangwal, S. Mithbavkar; Ann Occup. Hyg. 39(1) (1995) 115

[37] E. Chirila, I. Carazeanu, S. Dobrinas, Talanta, 53 (2000) 271.

38] M. Achilli, L. Romele, Journal of Chromatography A, 847 (1999) 271.

[39] L. Romele, M. Achilli, Analyst, 123 (1998) 291.

[40] J. F. Goodwin, Clinical Chemistry, 17(6) (1971) 544
[41] P. P W. West, J. K. Carlton, Analytical Chemistry, 27 (1949) 1055

[42] P. W. West, E. S. Amls, Industrial and Engineering Chemistry, 18(6) (1946), 400.

43] M. M. Kosanic, J. S. Trickovic, Journal of Photochemistry and Photobiology A: Chemistry, 149 (2002) 247.

[44] Vogel, A. Química Analítica Qualitativa, Mestre Jou, São Paulo, SP, 1981.

[45] P. K. Dasgupta, K. DeCesare, J. C. Ullrey, Anal. Chem.

$52(1980) 1912$

[46] HARRIS, D. C., Análise Química Quantitativa, LTC editora, Rio de Janeiro RJ, 2001.

47] SKOOG, D. A., WEST, D. M., HOLLER, F. J, Fundamentals of Analytical Chemistry, Ed. Harcourt College Publishing, 7ed, 1997. 